\title{
Bridging The Gap: Demanding 1-1 Representation of Dark-Light Skin Tones Within Medical Lectures/Resources
}

\section{Delice Kayishunge* $^{*}$ Mason Belue* $^{*}$}

University of Arkansas for Medical Sciences College of Medicine, Little Rock, AR

*Authors contributed equally

https://doi.org/10.38126/JSPG180412

Corresponding author: dkayishunge@uams.edu or mbelue@uams.edu

Keywords: skin of color; medical education; dermatology; ethnic; healthcare disparities; minority; socioeconomic

Executive Summary: Being a good physician means having the ability to recognize diseases in all kinds of individuals. This is especially true for skin lesions (e.g., acne, cancer), which present differently based on skin color and tone. Developing skin-tone-dependent diagnosing skills depends on the medical education (e.g., lectures, medical textbooks, and online board certification prep resources) and hands-on clinical experiences doctors receive. We find it alarming that medical students' gold standard resources overrepresent light skin and underrepresent dark skin to the point where many medical students can recognize a lesion on white skin but fail to recognize a similar lesion on dark skin. This lack of representation perpetuates race as a social determinant of health, leading to missed diagnoses and diagnosis at a later/worse stage in people of color. To combat this underrepresentation within medical education, we propose the Liaison Committee on Medical Education (LCME) amend Accreditation Standard 7: Curricular Content, Subsection 7.6: Cultural Competence and Health Care Disparities. The amendment is to include 1 of the 2 following policy changes, with preference for the top-down mandate: 1) Top-down Mandate: An objective measure and subsequent goal (1:1 representation) for the representation of skin of color within a school's medical lectures, which is evaluated by an LCME-approved curriculum committee and mandated for schools wishing to continue to be LCME accredited. 2) Bottom-up Individualized Institutional Goals: A requirement for schools to choose their own goal, create their committee, and evaluate their progress. These progress reports will be submitted to the LCME annually.

\section{Background}

Dermatological health disparities disproportionately affect patients with skin of color (SoC) leading to delayed treatment courses and increased morbidity and mortality, e.g., finding skin cancer too late and at a worse stage (Fourniquet et al. 2019, 06.18; Tripathi et al. 2018, 1286-91; Buster et al. 2012, 53-viii). Of the many factors that contribute to this disparity, one factor that is education-dependent and physiciandependent is a lack of familiarity with disease presentation in patients with SoC (Wang et al. 2015; Ezenwa et al. 2021). This lack of familiarity stems from a lack of representation of SoC within books that teach dermatology, resources for board exam preparation, and school lectures. This point is driven home by studies showing that medical students are less accurate in diagnosing dermatological conditions such as squamous cell carcinoma, atopic dermatitis, and urticaria in patients with SoC (Fenton et al. 2020, 957-58).

Our initial focus on dermatology textbooks revealed one study which analyzed 4,146 images from the most common atlases, including Atlas of Human Anatomy, Bates' Guide to Physical Examination \& History Taking, Clinically Oriented Anatomy, and Gray's Anatomy for Students by coding for a race (white, Black, and Person of Color) as well as skin 
tone (light, medium, dark). They found that although many of the textbooks approximate the racial distribution of the US population-62.5\% white, $20.4 \%$ Black, and $17.0 \%$ People of Color (POC) 一that they overrepresented light skin and underrepresented dark skin-74.5\% light, 21\% medium, and 4.5\% dark (Louie et al. 2018, 38-42; Ebede et al. 2006, 687-90). Additionally, there are numerous chapters where images on POC and dark skin are absent, such as for instances of skin cancer. This lack of representation throughout the dermatology textbooks contributes to the lack of familiarity of physicians, inevitably leading to missing important signs on dark-skinned patients because either they do not know what to look for or due to book chapters wrongly associating certain conditions like skin cancer only with the "high-risk" white population (Adelekun et al. 2021, 194-96; Louie et al. 2018, 38-42). Resources used in preparation for medical board examinations are no better. For instance, UWorld STEP 2 CK Qbank, UWorld Step 1 Qbank, Pathoma, and First Aid 2020, contain 516 unique images, of which only 125 images (24\%) are of SoC. The top conditions depicted in SoC include tinea infections (12\%), lichen planus (6.4\%), vitiligo $(6.4 \%)$, psoriasis $(5.6 \%)$, and erythema nodosum $(4.8 \%)$. Notably, these conditions are not among the most common skin conditions, such as Acne vulgaris or atopic dermatitis, and they also are not the ones that carry the most significant risk and disease burden such as skin cancer (Jones et al. 2020). While resources are deficient in the representation of SoC overall, this last point emphasizes that the represented areas are not the ones that should be focused on.

To summarize, SoC is not adequately incorporated into general dermatologic medical education, and when they are, they overrepresent light skin and underrepresent dark skin. This problem is widespread, affecting the most popular dermatology textbooks and the gold standards for board prep. The result is generations of medical students that lack familiarity with SoC perpetuating skin tone as a social determinant of health.

\section{Current legal/political outlook}

This nation has a history of centuries of unequal treatment and discrimination against POC, with inevitable consequences like lack of representation of dark skin in medical education. Over the past decade, we witnessed various organizations across the U.S. adopt policies to address racism, discrimination, and inequity. Generally, these policies demonstrate the significance of racial equity, inequity and discriminatory behavior, and a plan of action to achieve a non-discriminatory environment. We find it appropriate that the medical field should be particularly concerned with remedying the present effects of past political and legal discrimination.

According to the US Census Bureau projects, half of all patients will be individuals with SoC by 2050 (Colby et al. 2015, P25-1143). Since skin conditions present differently from one skin tone to another, doctors need to be preparing accordingly. Racial inequities in health care delivery rooted in the implicit bias created by images that do not reflect patients' racial demographics have been well documented. In their study, they assessed medical students' diagnostic accuracy using clinical images (Fitzpatrick IV-VI vs. Fitzpatrick I-III) (Fenton et al. 2020, 957-58). They found that nearly $34 \%$ of students misdiagnosed squamous cell carcinoma in SoC as melanoma. Those inaccuracies contribute to late-stage diagnoses and survival differences between patients with SoC and white patients.

The Liaison Committee on Medical Education (LCME) is an organization recognized by the US Department of Education to accredit medical schools in the United States and Canada. Generally, every eight years, LCME evaluates the quality of education provided at an institution and determines whether that institution and its students meet the twelve established standards. Although LCME oversees the effort to ensure medical schools broaden their students' gender, racial, cultural, and economic diversity, it is the medical schools which put in the groundwork. Medical schools themselves must develop programs that increase diversity, equity, and inclusion during recruitment, admission, and retention processes. They also must regularly assess how well their goals are being achieved. Additionally, the responsibility to prepare a medical curriculum that addresses medical consequences of common societal problems belongs to a medical school faculty. We want to emphasize that the accuracy with which doctors diagnose skin conditions on SoC depends on the exposure and the education received, specifically the images used during training. In the light of demographic trends, we recognize an absolute need for diversity in skin 
images used during medical school and propose an approach that we believe would be both realistic and effective.

\section{Option 1: Mandate (top-down)}

i. Amend the Liaison Committee on Medical Education (LCME) accreditation Standard 7: Curricular Content, subsection 7.6: Cultural Competence and Health Care Disparities

The amendment is to include an objective measure of the representation of skin of color within a school's medical lectures. These efforts will be measured by the percentage of medical photos shown for a disease/cutaneous manifestation with both light (less than or equal to III on the Fitzpatrick scale) and dark (greater than IV on the Fitzpatrick scale) skin. The goal ratio of light:dark should be as close to $1: 1$ representation as possible with a 0.2 standard deviation for improvement.

ii. Implement a curriculum committee at each LCME accredited medical school whose sole job is to continuously evaluate lecture/content material, and score the light:dark representation

This committee will also guide the faculty on which books/resources to use based on relevance and equal representation.

iii. Create a cross-institutional image database that LCME accredited medical schools must contribute to Images uploaded to this database are images of disease/cutaneous manifestations in light and dark skin.

\section{iv. Advantages}

- Directly incentivizes medical schools that want to maintain their LCME accreditation to represent light and dark skin tones within their curriculum.

- Allows faculty to submit current lectures to the committee, who then scores their lectures and makes specific recommendations on what to add/remove.

- Improves production and access of images across institutions.

v. Disadvantages

- Creates a high demand for dark skin images, which are not readily available/accessible to most institutions without subscriptions to dermatology image databases such as VisualDx.

- The new system could place people of color at a higher risk of breached confidentiality and coercion to let images be taken of their skin.

\section{Option 2: Individual institution goal driven (bottom-up)}

i. Instead of trying to match a national standard set by the LCME, LCME would encourage each school to identify its own goals and work towards them Each institution will evaluate its progress and adjust Option 2 ii-vi. Progress reports will be sent annually to the LCME on how well each institution meets its own goals. LCME will review changes made to the existing curriculum. Once approved, these change elements will become part of the compliance requirements for the medical education program. LCME will also make recommendations based on other schools' reporting and progress.

ii. Create a curriculum committee whose role is to audit lectures, PowerPoints, and books to ensure that the images shown represent existing demographics and do not perpetuate racial biases

\section{iii. As part of Continuing Medical Education, medical} schools will be encouraged to host workshops to teach health providers in hospitals and communities about skin of color.

Workshops could include history lessons on medical racism and violence against people of color in the medical school curriculum and implicit bias training.

iv. Medical schools will be encouraged to initiate a multi-department effort to collect photos and build a photo library representative of skin conditions on all skin shades

Safeguards must be in place to minimize the risk of harm and exploitation. Such safeguards include the proper obtainment of informed consent, assessment of decisional capacity in critically ill patients, and exclusion of patients incapable of making their own decisions.

\section{v. Advantages}

- Letting schools set their own goals allows for a more individualized approach.

- Graduating medical doctors who are adequately trained can mitigate their own 
unconscious biases and provide the best care for patients of all skin shades without misdiagnosis or delayed care.

- A new standard of education that genuinely addresses health care inequities and actively opposes racism.

\section{vi. Disadvantages}

- Although participation is required, it would be hard for the LCME to evaluate progress from institutions with varying goals.

- These interventions will require sustained efforts and time for them to result in permanent and long-term solutions.

- Potentially compromise patients' confidentiality.

- Additional training and resource expenditures might be a potential source of anger and frustration for some stakeholders as they may disrupt current curricular reform initiatives being undertaken by universities.

\section{Option 3: No change}

\section{i. Advantages}

- Maintaining the current system would result in no economic disturbances, saving time and money, which can be used in other programs.

\section{ii. Disadvantages}

- Continual use of textbooks and resources non-reflective of US demography equates to a failure to adapt to the demographic trends.

\section{References}

Adelekun, Ademide, Ginikanwa Onyekaba, and Jules B. Lipoff. 2021. "Skin Color in Dermatology Textbooks: An Updated Evaluation and Analysis." Journal of the American Academy of Dermatology 84 (1): 194-96. https://doi.org/10.1016/i.jaad.2020.04.084.

Buster, Kesha J., Erica I. Stevens, and Craig A. Elmets. 2012. "Dermatologic Health Disparities." Dermatologic Clinics 30 (1): 53-viii. https://doi.org/10.1016/j.det.2011.08.002.

Colby, Sandra L., and Jennifer M. Ortman. 2015. Projections of the Size and Composition of the U.S. Population: 2014 to 2060. Population Estimates and Projections. Current Population Reports. P25-1143. US Census Bureau. US Census Bureau. https://eric.ed.gov/?id=ED578934.
- Failure to live up to the medical maxim of "beneficence and nonmaleficence".

\section{Conclusion}

In the last decade, US institutions in all sectors have been taking steps and pledges against racism. Notably, every medical school claims "Diversity/Inclusion" as one of its core values in some shape or form. There are many ways to reduce racial and ethnic health disparities, but we believe the biggest of all is through education. The approaches described in Option 1 are what we recommend as the best solution, but Option 2 is a worthwhile consolation if Option 1 cannot be achieved. They will open the floodgate to many benefits, including but not limited to improved ability of medical students to identify skin lesions in the skin of color, prompt dermatology research within the field of the skin of color, physicians more consistently making the correct diagnosis early, reduction of patient referrals and their associated costs, improvement in hospital efficiency, and most importantly lead to better health outcomes for our patients of color. Our success in serving patients of all skin colors depends on the education we receive, and that is why we urge medical institutions and the LCME to stay true to their vision of reducing health inequalities and become dedicated to directly addressing the issue.
Ebede, Tobechi, and Art Papier. 2006. "Disparities in Dermatology Educational Resources." Journal of the American Academy of Dermatology 55 (4): 687-90. https://doi.org/10.1016/i.jaad.2005.10.068.

Ezenwa, Ekene, and Kesha Buster. "Health Disparities And Skin Cancer In People Of Color - Practical Dermatology". 2021. Practical Dermatology. https://practicaldermatology.com/articles/201 9-apr/health-disparities-and-skin-cancer-inpeople-of-color.

Fenton, Anne, Erika Elliott, Ashkan Shahbandi, Ekene Ezenwa, Chance Morris, Justin McLawhorn, James G. Jackson, Pamela Allen, and Andrea Murina. 2020. "Medical Students' Ability to Diagnose Common Dermatologic Conditions in Skin of Color." Journal of the American Academy of Dermatology 83 (3): 957-58. https://doi.org/10.1016/i.jaad.2019.12.078. 
Fourniquet, Sophie Elise, Kristen Garvie, and Kaylin Beiter. 2019. "Exposure to Dermatological Pathology on Skin of Color Increases Physician and Student Confidence in Diagnosing Pathology in Patients of Color." The FASEB Journal 33 (S1): 606.18-606.18. https://doi.org/10.1096/fasebj.2019.33.1_sup plement.606.18.

Jones, Virginia A., Kayla A. Clark, Morinola T. Shobajo, Adriana Cordova, and Maria M. Tsoukas. 2020. "Skin of Color Representation in Medical Education: An Analysis of Popular Preparatory Materials Used for United States Medical Licensing Examinations." Journal of the American Academy of Dermatology, August. https://doi.org/10.1016/i.jaad.2020.07.112.

"LCME Accreditation." AAMC. Accessed July 12, 2021. https://www.aamc.org/services/first-forfinancial-aid-officers/lcme-accreditation.
Louie, Patricia, and Rima Wilkes. 2018. "Representations of Race and Skin Tone in Medical Textbook Imagery." Social Science \& Medicine 202 (April): 38-42. https://doi.org/10.1016/j.socscimed.2018.02. $\underline{023}$.

Tripathi, Raghav, Konrad D. Knusel, Harib H. Ezaldein, Jeffrey F. Scott, and Jeremy S. Bordeaux. 2018. "Association of Demographic and Socioeconomic Characteristics With Differences in Use of Outpatient Dermatology Services in the United States." JAMA Dermatology 154 (11): 1286-91. https://doi.org/10.1001/jamadermatol.2018.3 114.

Wang, Stephanie, Lynda Shadrake, Pauline Scott, Hajwa Kim, and Claudia Hernandez. 2015. "Medical Student Melanoma Detection Rates in White and African American Skin Using Moulage and Standardized Patients." Clinical Research in Dermatology Open Access 2 (1). DOI: http://dx.doi.org/10.15226/2378$\underline{1726 / 2 / 1 / 00}$

Delice Kayishunge is a third-year medical student at the University of Arkansas for Medical Sciences (UAMS). She received her bachelor's degree in Biology from Lyon College and a master's degree in Information Quality from the University of Arkansas at Little Rock (UALR). Her clinical interest is in academic dermatology and the pursuit of health and social justice for all. Beyond research and medicine, she serves in a combat support hospital unit in the Army Reserves. Outside of educational and career interests, she enjoys spending time with her friends and loved ones, cooking, traveling, and exercising.

Mason Belue is a third-year medical student at the University of Arkansas for Medical Sciences (UAMS). Belue received their bachelor's degree in biomedical engineering from the University of Arkansas Fayetteville and wishes to pursue a career as a clinician-scientist with a focus on molecular imaging and artificial intelligence. 